\title{
Nanotechnology in fertilizers
}

To the Editor - Nitrogen, which is a key nutrient source for food, biomass, and fibre production in agriculture, is by far the most important element in fertilizers when judged in terms of the energy required for its synthesis, tonnage used and monetary value. However, compared with amounts of nitrogen applied to soil, the nitrogen use efficiency (NUE) by crops is very low. Between 50 and $70 \%$ of the nitrogen applied using conventional fertilizers - plant nutrient formulations with dimensions greater than $100 \mathrm{~nm}$ - is lost owing to leaching in the form of water soluble nitrates, emission of gaseous ammonia and nitrogen oxides, and long-term incorporation of mineral nitrogen into soil organic matter by soil microorganisms ${ }^{1}$. Numerous attempts to increase the NUE have so far met with little success, and the time may have come to apply nanotechnology to solve some of these problems.

Carbon nanotubes were recently shown to penetrate tomato seeds ${ }^{2}$, and zinc oxide nanoparticles were shown to enter the root tissue of ryegrass ${ }^{3}$ (Fig. 1). This suggests that new nutrient delivery systems that exploit the nanoscale porous domains on plant surfaces can be developed. The potential use of nanotechnology to improve fertilizer formulations, however, may have been hindered by reduced research funding and the lack of clear regulations and innovation policies. Current patent literature shows that the use of nanotechnology in fertilizer development remains relatively low (about 100 patents and patent applications between 1998 and 2008) compared with pharmaceuticals (more than 6,000 patents and patent applications over the same period) ${ }^{4}$.

A nanofertilizer refers to a product that delivers nutrients to crops in one of three ways. The nutrient can be encapsulated inside nanomaterials such as nanotubes or nanoporous materials, coated with a thin protective polymer film, or delivered as particles or emulsions of nanoscale dimensions. Owing to a high surface area to volume ratio, the effectiveness of nanofertilizers may surpass the most innovative polymer-coated conventional fertilizers, which have seen little improvement in the past ten years.

Ideally, nanotechnology could provide devices and mechanisms to synchronize the release of nitrogen (from fertilizers)

a

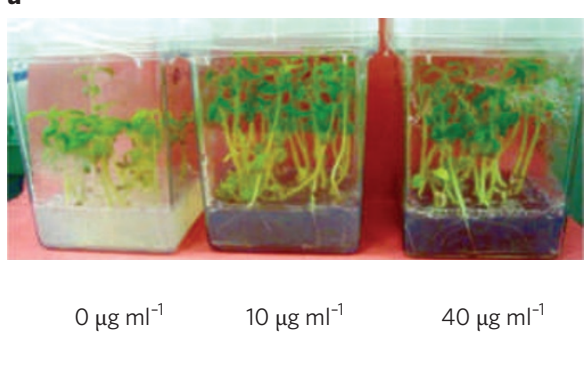

Concentration of nanotubes in growth medium

b

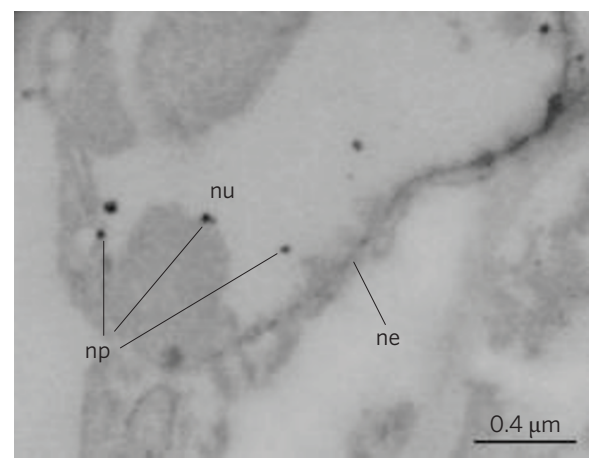

Figure 1 | Nanomaterials can enter plants. a, Improved growth of 27-day-old tomato seedlings grown in a medium containing carbon nanotubes. Reproduced with permission from ref. 2 (c) 2009 ACS). b, Transmission electron micrograph of ryegrass roots showing the entry of zinc oxide nanoparticles (nu, nucleus; ne, nuclear envelope; np, nanoparticles. Reproduced with permission from ref. 3 (c) 2008 ACS).

with its uptake by crops; the nanofertilizers should release the nutrients on-demand while preventing them from prematurely converting into chemical/gaseous forms that cannot be absorbed by plants. This can be achieved by preventing nutrients from interacting with soil, water and microorganisms, and releasing nutrients only when they can be directly internalized by the plant.

Examples of these nanostrategies are beginning to emerge. Zinc-aluminiumlayered double-hydroxide nanocomposites have been used for the controlled release of chemical compounds that regulate plant growth ${ }^{5}$. Improved yields have been claimed for fertilizers that are incorporated into cochleate nanotubes (rolled-up lipid bilayer sheets) ${ }^{6}$. The release of nitrogen by urea hydrolysis has been controlled through the insertion of urease enzymes into nanoporous silica ${ }^{7}$. Although these approaches are promising, they lack mechanisms that can recognize and respond to the needs of the plant and changes in nitrogen levels in the soil. The development of functional nanoscale films ${ }^{8}$ and devices has the potential to produce significant gains in the NUE and crop production.

In addition to increasing the NUE, nanotechnology might be able to improve the performance of fertilizers in other ways. For example, owing to its photocatalytic property, nanosize titanium dioxide has been incorporated into fertilizers as a bactericidal additive. Moreover, titanium dioxide may also lead to improved crop yield through the photoreduction of nitrogen gas ${ }^{9}$. Furthermore, nanosilica particles absorbed by roots have been shown to form films at the cell walls, which can enhance the plant's resistance to stress and lead to improved yields ${ }^{10}$.

Clearly, there is an opportunity for nanotechnology to have a profound impact on energy, the economy and the environment, by improving fertilizer products. New prospects for integrating nanotechnologies into fertilizers should be explored, cognizant of any potential risk to the environment or to human health. With targeted efforts by governments and academics in developing such enabled agriproducts, we believe that nanotechnology will be transformative in this field.

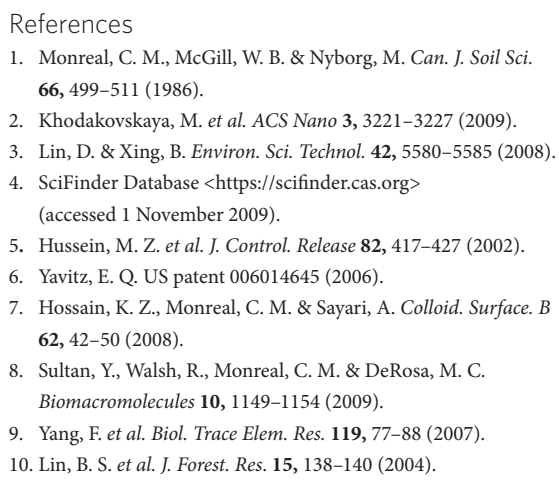

Maria C. DeRosa ${ }^{1 \star}$, Carlos Monreal ${ }^{2}$, Morris Schnitzer ${ }^{2}$, Ryan Walsh ${ }^{1}$ and Yasir Sultan ${ }^{1}$ 'Department of Chemistry, Carleton University, 1125 Colonel By Drive, Ottawa, Ontario K1S 5B6, Canada, ${ }^{2}$ Agriculture and AgriFood Canada, 960 Carling Avenue, Ottawa, Ontario K1A 0C6, Canada.

*e-mail:maria_derosa@carleton.ca 